DESY 09-163

\title{
Higgs production as a probe of Chameleon Dark Energy
}

\author{
Philippe Brax ${ }^{1}$, Clare Burrage ${ }^{2}$, Anne-Christine Davis ${ }^{3}$, \\ David Seery $^{3}$ and Amanda Weltman ${ }^{3,4,5}$
}

${ }^{1}$ Institut de Physique Théorique, CEA, IPhT, CNRS, URA2306, F-91191

Gif-sur-Yvette cédex, France

2 Theory Group, Deutsches Elektronen-Synchrotron DESY, D-22603, Hamburg, Germany

${ }^{3}$ Department of Applied Mathematics and Theoretical Physics, Centre for Mathematical Sciences, University of Cambridge, Wilberforce Road, Cambridge, CB3 0WA, United Kingdom

${ }^{4}$ Department of Mathematics and Applied Mathematics, University of Cape Town, Private Bag, Rondebosch, South Africa, 7700

${ }^{5}$ Centre for Theoretical Cosmology Fellow

E-mail: philippe.brax@cea.fr, clare.burrage@desy.de, A.C.Davis@damtp.cam.ac.uk, djs61@cam.ac.uk, amanda.weltman@uct.ac.za

\begin{abstract}
In this paper we study various particle physics effects of a light, scalar dark energy field with chameleon-like couplings to matter. We show that a chameleon model with only matter couplings will induce a coupling to photons. In doing so, we derive the first microphysical realization of a chameleonic dark energy model coupled to the electromagnetic field strength. This analysis provides additional motivation for current and near-future tests of axion-like and chameleon particles. We find a new bound on the coupling strength of chameleons in uniformly coupled models. We also study the effect of chameleon fields on Higgs production, which is relevant for hadron colliders. These are expected to manufacture Higgs particles through weak boson fusion, or associated production with a $Z$ or $W^{ \pm}$. We show that, like the Tevatron, the LHC will not be able to rule out or observe chameleons through this mechanism, because gauge invariance of the low energy Lagrangian suppresses the corrections that may arise.
\end{abstract}

Keywords: Dark energy theory, Weak interactions beyond the Standard Model, Cosmology of theories beyond the Standard Model

PACS codes: 95.36.+x; 12.15.-y, 14.80.Va, 98.80.-k 


\section{Introduction}

We now have compelling evidence that the rate of expansion of the universe is accelerating, requiring the universe to be dominated by an unknown form of matter, known as 'dark energy,' characterized by the equation of state $p \approx-\rho$. This dark energy could be a cosmological constant unnaturally tuned at the level of 1 part in $10^{123}$ [1], or it may be associated with the potential energy of a scalar field [2, 3, 4, 5]. If a scalar field drives the accelerated expansion then it must be extremely light, with mass of order $H_{0} \sim 10^{-33} \mathrm{eV}$. The existence of light scalar fields results in new, long range "fifth forces," which are tightly constrained by experiment [6]. To avoid these constraints the energy scale controlling the coupling of such scalar fields to matter must be many orders of magnitude larger than the Planck scale [5]. Explaining why this coupling is so weak is a major problem for dynamical dark energy models.

These problems can be circumvented by allowing the scalar field mass mass to be determined by a chameleon mechanism [7, 8, 9, 10, 11]. This makes the field heavy in a dense environment, but light in vacuum. Such scalar fields hide from experimental searches for fifth forces [7, 8] and modifications of gravity [12, 13] in a novel way: in the interior of a massive object the chameleon is very heavy, and has a correspondingly short interaction length. An observer outside the body feels a scalar force sourced only by a thin shell of matter at the surface of the object. This suppresses unwanted fifth forces [7, 8]. Observations impose few constraints on the strength of the coupling of the scalar field to the Standard Model, allowing the energy scale of the coupling to be many orders of magnitude below the Planck scale [11].

Chameleonic scalar fields have been shown to have a successful classical phenomenology. Their equation of state depends on the energy density of the universe. In the early universe the chameleon behaves as an additional matter component, but at late times it has an equation of state with roughly $w \approx-1$. This enables it to drive an era of accelerated expansion [14. Weak constraints on the initial conditions ensure that the chameleon does not disrupt the dynamics of the early universe [14, 11]. In this paper we discuss these theories in the framework of quantum mechanics. This is of interest because the cosmological constant problem is essentially quantum mechanical. There is no profit in replacing the cosmological constant by some other theory which is equally unnatural once quantum corrections are taken into account.

The chameleon mechanism makes it mandatory for the dark energy scalar field to interact with conventional matter, and these interactions are potentially very strong. They may help us unravel the microphysics of dark energy if their indirect consequences could be measured [15, 16, 17, 18, 19, 20, 21]. If the interactions are sufficiently strong, however, then an unambiguous direct consequence may be observable: new, light scalar quanta must be present in the beam pipe of any particle accelerator, raising the prospect of observing dark energy in the laboratory. Fully consistent quantum mechanical realizations of the chameleon have not yet been constructed, and may suffer from the same naturalness and tuning difficulties as quintessence [22, 23, 24]. In this paper we do 
not attempt to address naturalness concerns, or the quantum-mechanical construction of the model. Instead, supposing such models to exist, we determine constraints which are imposed by experiment.

Real or virtual chameleon-like particles will certainly be produced in particle collisions if dark energy couples to the electroweak gauge bosons. In this paper, we will provide a microscopic derivation of this coupling for any chameleon-like model. The models which furnish chameleon-like scalar particles at low energy are effective theories, valid below some energy scale $M_{c}$. In Ref. [25], corrections to electroweak precision observables from particles with chameleonic or axionic couplings were analysed, without making a commitment to any specific choice of physics at energies above $M_{c}$. It was shown that very weak constraints on the energy scale of the chameleon coupling to matter ensure that the chameleon does not have an observable effect on measurements of the $Z$-width. For processes involving fermions and $\mathrm{SU}(2) \times \mathrm{U}(1)$ gauge bosons, it was shown that large effects could always be absorbed into renormalizations of the Fermi constant, $G_{F}$, and the gauge boson masses. When Higgs processes are included it is no longer clear that large effects can be hidden in this way, potentially allowing Higgs production to function as a diagnostic of dark energy physics and its coupling to the Standard Model.

In this paper, we focus on the consequences of such dark energy quanta for Higgs production. This is a key target for both the Large Hadron Collider (LHC) and the Tevatron. The details of Higgs production depend on which couplings occur in the theory. To achieve a successful chameleon phenomenology we assume conformal couplings to matter $\dagger$ This has implications for interactions with gauge boson kinetic terms, which will be described in more detail in \$4 below. The Standard Model gauge bosons have conformally invariant kinetic terms and develop no couplings to a scalar field of this type. In this paper we show that violations of conformal symmetry, arising from couplings to matter species, can be communicated to the kinetic term by quantum corrections. Therefore, a coupling is generated at energies below $M_{c}$ via loops of charged heavy particles. This coupling has important phenomenological consequences which will be briefly recalled. In particular we will show that this coupling induces a coupling to the electromagnetic field and in so doing provides a motivation for the effects probed in quantum laser experiments [28, 29, 30, 31, 32, 20, 21]. Our results apply for any light scalars with the requisite chameleon-like couplings, whatever their origin.

What scale, $M_{c}$, should be associated with these new degrees of freedom? A conservative choice would be the GUT scale, $M_{c} \sim 10^{16} \mathrm{GeV}$, which may be connected with an early inflationary stage, or a seesaw explanation of neutrino mass [33, 34, 35]. If so, heavy neutrinos of mass $M_{c}$ might exist but would be sterile, having no $\mathrm{SU}(2) \times \mathrm{U}(1)$ quantum numbers. This would not lead to the required coupling. Alternatively, if supersymmetry is realized in nature then $M_{c}$ could be associated with the scale at

$\dagger$ All chameleon models developed to date have conformal couplings to matter. However there exist related models of scalar fields with environment dependent properties which do not require conformal couplings [26, 27. 
which it is spontaneously broken, perhaps of order 1-10 TeV. In any supersymmetric completion of the Standard Model there exist fermionic partners of $\gamma, W^{ \pm}, Z$ and the Higgs, known as gauginos and Higgsinos. (For a review see [36] and references therein.) The mass eigenstates of these particles ("charginos") would naturally be of order $M_{c}$. In this article we remain agnostic about the nature of whatever particles circulate within loops. We give our calculation in a form which can be specialized immediately to the minimal supersymmetric Standard Model (MSSM), but our conclusions are general and do not depend on the details of a specific implementation. In any case, the calculation we describe can be adapted easily to any heavy particle carrying the requisite quantum numbers.

The outline of this paper is as follows. In \$2 we introduce our model and show that a coupling between gauge bosons and dark energy is generated at low energy by integrating out heavy particles. Some of the phenomenology associated with this coupling is presented. In $\S 3$ we briefly review Higgs production at a hadron collider, emphasizing the role of vertices between the electroweak gauge bosons and the Higgs. In $\$ 4$ we compute corrections to the Higgs production rate arising from the low energy theory written down in $\$ 2$. We show that the effective Lagrangian includes new contact interactions between the gauge bosons and the Higgs. At scales smaller than $1 / M_{c}$ these contact interactions resolve into heavy particle loops. We compute the correction to Higgs production from both effects. In $\$ 5$ we conclude by arguing that the effect of chameleon-like particles on Higgs production is expected to be rather small.

Throughout this paper, we adopt units in which $c=\hbar=1$. We set the reduced Planck mass, $M_{\mathrm{P}} \equiv(8 \pi G)^{-1 / 2}$, to unity. Our metric convention is $(-,+,+,+)$.

\section{A low-energy theory of gauge bosons and scalars}

Khoury \& Weltman [8, 7] suggested that a scalar field, $\chi$, might evade detection and yet remain light in vacuum if it coupled to the matter species, $\psi^{i}$, via a set of conformally rescaled metrics

$$
g_{a b} \rightarrow g_{a b}^{\alpha}=f_{\alpha}\left(\beta_{\alpha} \chi\right) g_{a b} .
$$

In this formula, $g_{a b}$ is the spacetime metric, and $\beta_{\alpha} \equiv 1 / M_{\alpha}$ is a coupling scale that is not necessarily related to the cutoff controlling the validity of the theory, $M_{c}$. The Einstein frame action is

$$
S=\int \mathrm{d}^{4} x \sqrt{-g}\left(\frac{M_{P}^{2}}{2} R-\frac{1}{2}(\partial \chi)^{2}-V(\chi)\right)-\int \mathrm{d}^{4} x \mathcal{L}_{m}\left(\psi^{i}, g_{a b}^{\alpha}\right),
$$

$\dagger$ If the different matter species do not interact, then each species can be chosen to couple to $\chi$ through a single metric. In this case, the indices $i$ and $\alpha$ are the same and the species $\psi^{i}$ couples to the metric $g_{a b}^{i}$ only. Where interactions among the matter species are present, each species may couple to more than one metric.

Alternatively, if $\chi$ couples to matter through a species-independent conformal rescaling of the metric, then the strength of the coupling to each matter species is the same. For phenomenological purposes, however, we wish to relax this restriction. In the remainder of this paper we allow the matter couplings to be distinct, but frequently return to the minimal scenario where all coupling strengths are the same. 
where $\mathcal{L}_{m}$ is the Lagrangian describing the dynamics of the matter fields, $\psi^{i}$. The dynamics of $\chi$ are controlled by an effective potential $V_{\text {eff }}$, which depends explicitly on the environment through $\mathcal{L}_{m}$ and satisfies

$$
V_{\mathrm{eff}}=V(\phi)+\frac{1}{2} \sum_{\alpha} f_{\alpha}^{2}\left(\beta_{\alpha} \chi\right) \frac{g_{a b}^{\alpha}}{\sqrt{-g^{\alpha}}} \frac{\delta \mathcal{L}_{m}\left(\psi^{i}, g_{a b}^{\beta}\right)}{\delta g_{a b}^{\alpha}},
$$

where the sum is over all metrics and $g^{\alpha}=\operatorname{det} g_{a b}^{\alpha}$. The conformal functions $f_{\alpha}$ and the potential $V$ must be chosen to realise a successful chameleon mechanism. This requires that the effective potential has a minimum. Another requirement is the existence of a thin shell effect [8], which is realized if the scalar field potential and coupling functions are chosen in such a way that large variations in the mass of the field can occur as the matter content of the local environment changes. Classical realizations of this idea have been constructed, but it is not known whether quantum realizations exist, or what condition the $f_{\alpha}$ must satisfy once quantum corrections are taken into account. In what follows we will neglect the indices $i$ and $\alpha$ for simplicity, but it is not necessary to assume that the dark energy field couples with the same strength to all matter fields.

We work in the Einstein frame, in which the gravitational part of the action is that of general relativity. If the scalar field couples conformally with universal strength, then a classically equivalent description can be obtained by transforming to the Jordan frame in which all effects of the scalar field reappear in the gravitational sector. In the classical theory, physical observables are independent of this choice. In the quantum theory, however, a non-trivial Jacobian may be necessary to connect the Einstein- and Jordanframe measures in the path integral [24]. In this paper we work in the Einstein frame from the outset, and neglect terms arising from the Jacobian. These make additional contributions to the coupling between gauge-boson kinetic terms and the dark energy scalar. We hope to return to this question in a future publication.

Despite appearances, chameleonic couplings of the form (11) do not necessarily give rise to large variations in fundamental constants or particle masses [14, 11]. This follows because the minimum of the effective potential is a cosmological attractor. The location of the minimum drifts only slowly, owing to time evolution of the matter density. This implies that variations in the scalar-dependent particle masses and fundamental constants are correspondingly small.

The kinetic terms of spin-1 particles are conformally invariant, and are left inert under the substitution $g_{a b} \rightarrow g_{a b}^{\alpha}$ in Eq. (1). However, particle physics is not conformal; any coupling to other matter species will generically break this invariance. If so, quantum corrections will cause the kinetic terms to depend on $g_{a b}^{\alpha}$. In this section we compute these threshold corrections, leading to an effective theory which describes the interaction of gauge bosons and the dark energy field at low energy. 


\subsection{Axion-like couplings from heavy particle loop $\$$}

The necessary corrections are depicted in Fig. 1, in which a heavy fermionic particle circulates in the loop. Fermions couple to the metric (11) via a vielbein, $e_{a}^{\mu}$, which satisfies

$$
g_{a b}=\eta_{\mu \nu} e_{(a}^{\mu} e_{b)}^{\nu} .
$$

There is an inverse vielbein, $e_{\mu}^{a}$, satisfying $e_{a}^{\mu} e_{\nu}^{a}=\delta_{\nu}^{\mu}$ and $e_{a}^{\mu} e_{\mu}^{b}=\delta_{b}^{a}$. The indices $\mu, \nu, \ldots$ transform under a rigid Lorentz symmetry and can be coupled to Dirac $\gamma$ matrices. The action for a Dirac fermion, $\lambda$, with large mass $M$, can be written

$$
L=\sqrt{-g} f^{2}(\beta \chi)\left\{-\bar{\lambda}\left(\gamma^{\mu} e_{\mu}^{a} D_{a}+M\right) \lambda\right\},
$$

where $D_{a}$ is a gauge-covariant derivative and $\bar{\lambda} \equiv \lambda^{\dagger} \gamma^{0}$ is the spinor conjugate to $\lambda$. We suppose that $\lambda$ transforms under an Abelian symmetry with gauge coupling constant $e$, so that

$$
D_{a} \equiv \partial_{a}+\frac{1}{8} \gamma_{\mu \nu} \omega_{a}^{\mu \nu}-\mathrm{i} e A_{a}
$$

where $\gamma_{\mu \nu}=\left[\gamma_{\mu}, \gamma_{\nu}\right]$. Note that the spin connexion, $\omega_{a}^{\mu \nu}$, transforms non-trivially under conformal rescalings. The calculation will be generalized to non-Abelian symmetries below Eq. (9). Conformal invariance is broken by the fermion mass, $M$. Like the mass of any canonical field, $M$ varies under conformal transformations like $M \rightarrow f^{1 / 2}(\beta \chi) M$. If all energy scales ran in the same way with the vacuum expectation value of the scalar field then the effects of this coupling would never be observable, but this is not the case. Both the Planck scale, $M_{P}$, and the scale controlling the strength of the scalar coupling to matter, $\beta$, are unchanged by variations in the scalar field.

We take the dark energy scalar to have a spatially-independent vacuum expectation value $\langle\chi\rangle=\bar{\chi}$, around which we quantize small fluctuations $\delta \chi$. (For this reason our calculation cannot be applied to very large spacetime volumes in which $\chi$ may develop appreciable gradients.) After rescaling the fermion fields to have canonical kinetic terms at leading order, the interaction between $\lambda$ and these fluctuations can be written

$$
L_{\mathrm{eff}} \supseteq-\frac{1}{2} \sqrt{-g} \frac{\bar{f}^{\prime}}{\bar{f}} \beta \mathcal{M}(\bar{\lambda} \lambda) \delta \chi,
$$

where $\bar{f} \equiv f(\beta \bar{\chi})$ and we have defined a conformally transformed mass $\mathcal{M} \equiv \bar{f}^{1 / 2} M$. Nevertheless, we emphasize that the physical mass of the fermion is $M$. A prime ' denotes the derivative of a function with respect to its argument.

In each diagram of Fig. 1, operators $A_{a}(q), A_{b}(q)$ and $\delta \chi(r)$ are inserted on the external legs, with all momenta flowing inwards. Fig. 1(b) depicts the "crossed" diagram, which corresponds to reversing the sense of momentum flow in the fermion loop. It can be obtained from Fig. 1(a) by the simultaneous replacements $p \leftrightarrow q$ and $a \leftrightarrow b$. Accounting for both diagrams we find that the correlation function $\left\langle A_{a}(q) A_{b}(p) \delta \chi(r)\right\rangle$

$\ddagger$ We would like to thank D. Shaw for very helpful discussions while preparing the text of this section. 

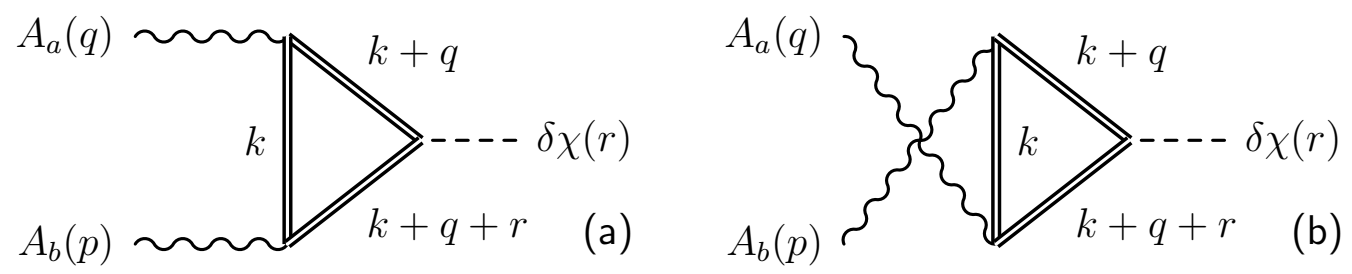

Figure 1. Diagrams contributing to the leading interaction between dark energy and the electroweak gauge bosons, which determine an effective operator acting on $A_{a}(q) A_{b}(p) \chi(r)$. Note that the momentum carried by $\chi$ is taken to flow into the diagram. Double lines represent a species of heavy fermion charged under $\mathrm{SU}(2) \times \mathrm{U}(1)$.

can be written

$$
\begin{aligned}
& \left\langle A_{a}(q) A_{b}(p) \delta \chi(r)\right\rangle=-(2 \pi)^{4} \delta(p+q+r) \frac{\bar{f}^{\prime}}{\bar{f}} \frac{e^{2} \beta \mathcal{M}}{2} \cdot \delta \chi(r) \cdot A_{a}(q) A_{b}(p) \times \\
& \int \frac{\mathrm{d}^{4} k}{(2 \pi)^{4}} \operatorname{tr}\left\{\gamma^{a} \frac{-\mathrm{i}(\not k+\not q)+\mathcal{M}}{(k+q)^{2}+\mathcal{M}^{2}-\mathrm{i} \epsilon} \frac{-\mathrm{i}(\not k-\not p)+\mathcal{M}}{(k-p)^{2}+\mathcal{M}^{2}-\mathrm{i} \epsilon} \gamma^{b} \frac{-\mathrm{i} \not k+\mathcal{M}}{k^{2}+\mathcal{M}^{2}-\mathrm{i} \epsilon}\right\} \\
& +\left(\begin{array}{c}
p \leftrightarrow q \\
a \leftrightarrow b
\end{array}\right),
\end{aligned}
$$

where 'tr' denotes a trace over Dirac indices.

The $k$ integral is divergent, and such integrals are not guaranteed to be invariant under the rigid shift $k^{a} \rightarrow k^{a}+a^{a}$. In the absence of a shift symmetry, the integral can depend on the labeling of momenta within the loop. We apply dimensional regularization to maintain gauge invariance, after which it can be checked that the result is insensitive to the routing of momentum through the diagram. Eq. (8) also contains a potentially gauge-violating zero derivative term, which would be proportional to $\delta \chi A^{a} A_{a}$ in real space. This term vanishes when the $k$ integral is evaluated using any gauge-invariant regulator. Discarding terms which are proportional to the equations of motion, we find that Eq. (8) can be reproduced from an effective Lagrangian of the form

$$
L_{\mathrm{eff}} \supseteq \frac{e^{2} \beta}{96 \pi^{2}} \frac{\bar{f}^{\prime}}{\bar{f}} \delta \chi F^{a b} F_{a b}+\mathrm{O}\left(\partial^{4}\right),
$$

where $\mathrm{O}\left(\partial^{4}\right)$ denotes terms containing four or more derivatives. Eq. (9) applies for each species of heavy fermion in the theory. If there are many such fermions, each contributes with its own $\beta$ and $f$. If $e, \bar{f}$ and $\bar{f}^{\prime}$ are of order unity for all species, the coupling will be dominated by the fermion with largest $\beta$. Similar couplings would be induced by scalar particles, such as heavy sleptons.

The physical effect of the coupling in Eq. (9) could also be understood in the Jordan frame. There, one would typically neglect the effect of dark energy because of its tiny coupling, of order $M_{\mathrm{P}}^{-1}$. In the model we are considering, this neglect would be unjustified. The scalar field is more strongly coupled, and its effects would be manifest in the curvature of spacetime caused by particles participating in an interaction. The curvature scale would be associated with energies $M \ll M_{\mathrm{P}}$, which would be larger than that typically associated with gravitational phenomena. 
In this calculation, the gauge field $A_{a}$ was taken to be Abelian. However, it is clear that the same calculation generalizes immediately to non-Abelian fields for which the heavy fermion, $\lambda$, transforms in the fundamental representation. Consider any gauge group with generators $t_{\alpha}$, so that $A_{a}=A_{a}^{\alpha} t_{\alpha}$. The $t_{\alpha}$ may be normalized to satisfy

$$
\operatorname{Tr}\left(t_{\alpha} t_{\beta}\right)=c_{1} \delta_{\alpha \beta},
$$

for an arbitrary constant $c_{1}$, given that ' $\operatorname{Tr}$ ' denotes a trace over indices in the gauge group. Eq. (9) therefore applies equally in a non-Abelian theory after the substitution $F^{a b} F_{a b} \rightarrow \operatorname{Tr} F^{a b} F_{a b}$.

Eq. (9) will be accompanied by more complicated corrections which couple $\operatorname{Tr} F^{a b} F_{a b}$ to all powers of $\delta \chi$. Resumming this expansion, we generate a coupling of the form

$B\left(\beta_{\gamma} \chi\right) \operatorname{Tr} F^{a b} F_{a b}$ for some function $B$ and coupling scale $\beta_{\gamma}$. Eq. (9) allows us to estimate the scale $\beta_{\gamma}$,

$$
\beta_{\gamma} \equiv \frac{e^{2} \beta}{96 \pi^{2}} .
$$

In the following we will assume, for simplicity, that no coupling to gluons is generated. The coupling to electromagnetism will give a small scalar field dependent contribution to the mass of atoms. If the theory were not of the chameleonic type, this would potentially lead to a strong violation of the weak equivalence principle. For chameleons, this will be heavily suppressed by the thin shell mechanism.

\subsection{Constraints on Chameleon couplings}

Eq. (9) shows that, in a conformally-coupled theory, even if contact interactions involving $\operatorname{Tr} F^{a b} F_{a b}$ are not present at high energies, they will inevitably be generated after passing the mass threshold of any matter species which couples both to $\chi$ and the gauge field. Therefore, laboratory and astrophysical bounds cannot be evaded merely by taking the $\delta \chi \cdot \operatorname{Tr} F^{a b} F_{a b}$ interaction to be absent, although their interpretation becomes model dependent. We believe this to be the first microphysical derivation of the coupling in Eq. (9).

This coupling means that chameleons are also axion-like particles: they couple to photons in an analogous way to the Peccei-Quinn axion, and therefore have a similar phenomenology. They are only 'axion-like' because the mass and couplings of the field are not related as they are for a standard axion, and because we are considering a scalar (rather than pseudo-scalar) field. Observational constraints on the couplings of axionlike particles are very tight. However, applying these constraints to chameleonic fields is not straightforward because the mass of the chameleon field depends on its environment. The strongest constraints on axion-like particles come from their production in the cores of stars, but a chameleon field becomes very massive in the interior of the sun and therefore its production through scattering processes is suppressed. The constraints on chameleons from such observations are discussed in more detail in Ref. [37]. For similar reasons chameleonic fields are not constrained by so-called "light shining through walls" searches for axion-like particles [38]. This is because the chameleon becomes heavy 
in the wall and is reflected by it rather than passing though. On the other hand, constraints that come from observing the behaviour of axion-like particles purely in diffuse environments can be applied to chameleonic fields. Strong constraints come from laboratory [31, 32, 20, 39] and astrophysical searches [29, 28, 40, 37, 41, 42]. Indeed, it is possible that certain astronomical observations may be explained most simply by including light scalars which couple as chameleons [43].

Under certain circumstances it is possible to translate the stringent bounds obtained from electromagnetic probes, discussed above, into bounds on the matter coupling. We assume a minimal model in which the dark energy couples to matter with a uniform strength $\beta$, irrespective of species. This coupling is subject to only mild restrictions, depending on the precise self-interaction potential which is chosen for the chameleon field. Even where such restrictions exist, they typically require $\beta$ to be no smaller than the ordinary scale of nuclear physics, $\beta \lesssim(100 \mathrm{GeV})^{-1}$ [11]. For $\beta \bar{\chi} \lesssim 1$ and gauge coupling $e \approx 0.5$, which is roughly the scale of the $\mathrm{SU}(2)$ and $\mathrm{U}(1)$ couplings of the Standard Model, we find

$$
\beta_{\gamma} \approx 10^{-4} \beta .
$$

Therefore, constraints on $\beta_{\gamma}$ can be translated to limits on the matter coupling, $\beta$. Unfortunately this constraint is highly model-dependent, and can be weakened arbitrarily by decreasing the gauge coupling $e$. Strong constraints are obtained only when $e$ can be determined by other means.

The strongest bound on $\beta_{\gamma}$ follows from observations of the polarization of starlight in the Milky Way, yielding $\beta_{\gamma} \lesssim\left(10^{9} \mathrm{GeV}\right)^{-1}$ for models in which the mass of the field satisfies $m_{\chi} \lesssim 10^{-11} \mathrm{eV}$ in the interstellar medium [37]. $\$$ In models where Eq. (12) applies, it follows that there is a new, stronger bound on the matter coupling $\llbracket$

$$
\beta \lesssim \frac{1}{10^{5} \mathrm{GeV}} \simeq \frac{10^{14}}{M_{\mathrm{P}}} .
$$

If the strength of the coupling of the scalar field to the gauge bosons is strong, we might also expect to see the scalar field in particle colliders. Because the chameleonic field is light in the vacuum of a particle collider it cannot be integrated out of the theory. In Ref. 25] the effects of dark energy on precision electroweak observables were discussed. Corrections from processes with scalar fields in the final state were shown to contribute only if the scale of the coupling is low. The best constraints come from observations of the width for $Z$-decay, and corrections to this from the scalar field are invisible if $\beta_{\gamma} \lesssim\left(10^{2} \mathrm{GeV}\right)^{-1}$. It was also shown that large corrections due to the scalar field are screened in all $2 \rightarrow 2^{\prime}$ fermion scattering interactions due to a combination of gauge invariance and the structure of boson/lepton couplings in the Standard Model.

$\S$ Constraints on the coupling of dark energy to photons, $\beta_{\gamma} \lesssim\left(10^{6} \mathrm{GeV}\right)^{-1}$, also come from the PVLAS [28] and GammeV [20] experiments, and apply to models where the field is sufficiently light in the vacuum tube and sufficiently heavy in the walls of the tube to prevent it escaping from the experiment.

$\|$ Consistency with our assumptions requires $\beta \bar{\chi} \lesssim 1$, corresponding to $\bar{\chi} \lesssim M_{\mathrm{P}} / 10^{10}$, which should be easily satisfied for the chameleon field in all relevant backgrounds. 

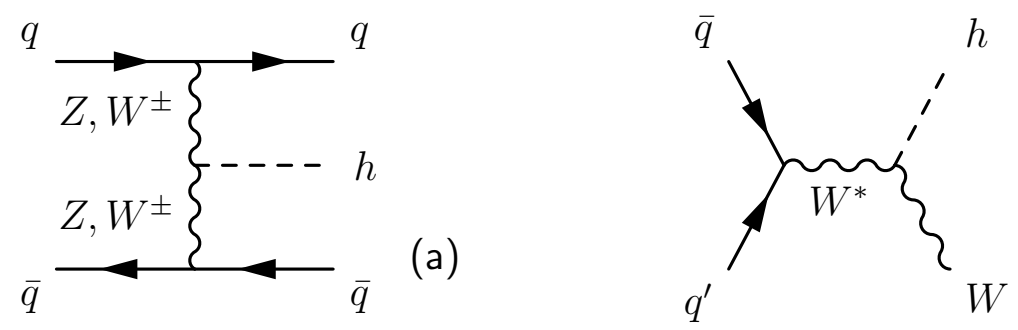

Figure 2. Higgs production channels. In (a), weak boson fusion is the next-to-leading process at a hadron collider, but benefits from accurate background subtraction. A $q \bar{q}$ pair undergoes the splitting $q \bar{q} \rightarrow q \bar{q} V \bar{V}$, where $q$ is a generic quark species and $V$ is a vector boson. The final state Higgs is radiated via fusion of the intermediate $V \bar{V}$ pair. In (b), associated production occurs when two quarks, $q^{\prime}$ and $\bar{q}$, fuse to form an off-shell $W$. The final state is achieved by Higgsstrahlung radiation. At a lepton collider the initial state $q^{\prime}, \bar{q}$ can be replaced by an $e^{+} e^{-}$pair and an intermediate $Z^{*}$. The final state Higgs is produced in association with an on-shell $Z$.

The only processes in the electroweak sector to which this screening theorem does not apply are those involving Higgs bosons. For this reason, we might wonder whether coupling a dark energy scalar to the Higgs boson would lead to a large enhancement of Higgs production in particle colliders. In the next section we will show that this loophole can be closed, and that one can expect all electroweak processes to be screened from chameleon corrections.

\section{Higgs production}

\subsection{Production at particle colliders}

Below the electroweak symmetry breaking scale of order $1 \mathrm{TeV}$, interactions of the lightest neutral Higgs, $h$, with the $Z$ boson are described by cubic and quartic couplings [44,

$$
L_{Z Z h}=\int \mathrm{d}^{4} x Z_{a} Z^{a}\left\{\sqrt{2^{1 / 2} G_{F}} M_{Z}^{2} h+\sqrt{2} G_{F} M_{Z}^{2} h^{2}\right\},
$$

The $Z$ mass is $M_{Z} \simeq 91.2 \mathrm{GeV}$ and the Fermi constant, $G_{F}$, is measured experimentally to be $G_{F} \simeq 1.17 \times 10^{-5} \mathrm{GeV}^{-2}$ [45]. Eq. (14) is numerically correct for a minimal Standard Model Higgs. In a two Higgs doublet model these couplings are shared between the two neutral scalars, leading to suppression by a numerical factor $\uplus$ Analogous interactions for the $W^{ \pm}$are obtained by the substitutions $Z_{a} Z^{a} \rightarrow 2 W_{a}^{+} W^{-a}$ and $M_{Z} \rightarrow M_{W}$.

A summary of the methods for Higgs production at a hadron collider can be found in the recent book by Kilian [46]. (See also Ref. [47].) One especially important production mechanism is 'weak boson fusion,' $V \bar{V} \rightarrow h$, shown in Fig. 2(a), where $V$ is any of the

$\dagger$ In a supersymmetric Standard Model this factor is $\sin (\beta-\alpha)$, where $\beta$ parametrizes the ratio of Higgs vacuum expectation values, $\tan \beta \equiv v_{2} / v_{1}$, and $\alpha$ is an angle which occurs when diagonalizing the Higgs mass matrix. See, eg., Ref. [36. In a simple MSSM, $\sin (\beta-\alpha)$ may be near unity. 
vector bosons $W^{ \pm}, Z$. This process is generally sub-dominant to the rate of gluon-gluon fusion, but benefits from more accurate background subtraction. The precursor bosons originate within spectator quarks, as shown in Fig. 2(a) and discussed in more detail in $\$ 3.2$ below. Following a boson fusion event, the spectator quarks are disrupted and initiate transverse hadronic jets.

Another interesting mechanism is 'associated production,' shown in Fig. 2(b), in which two quarks fuse to form a $W$ resonance that subsequently decays via "Higgsstrahlung" into an on-shell $W$ and a Higgs, $q^{\prime} \bar{q} \rightarrow W^{*} \rightarrow W h$. Then its primary decays will be to $b \bar{b}$ pairs. Weak boson fusion and associated production rely on couplings of the Higgs to two vector bosons. These vertices are present in the Standard Model and in many theories of beyond-the-Standard-Model physics, although in certain cases they can be tuned to be absent. In this section, we study the possibility that corrections due to dark energy can change the relationship of these vertices to the other measurable parameters of the Lagrangian. Our analysis applies for a large class of models in which these two-boson couplings are represented by Eq. (14) and its generalization to $W^{+} W^{-}$ interactions.

\subsection{The effective $W$ approximation.}

The processes of weak boson fusion and associated production can be studied using conventional perturbation theory, but alternative methods exist. An especially useful tool is the effective $W$ approximation (see, eg., Ref. [46]). For weak boson fusion, this means that the vector boson precursors are taken to be on-shell partons within colliding hadrons. In the parton picture, a hadron consists of three valence quarks which are surrounded by a sea of virtual particles. A probe which samples this sea at sufficiently high resolution has a chance to resolve the virtual quanta, rather than valence quarks. Since quarks participate in the electroweak interaction, $Z$ and $W^{ \pm}$bosons will be found within the virtual sea. Its precise composition can be determined by solving a system of DGLAP-like equations, which can be thought of as an approximate Boltzmann hierarchy [48]. In this picture, calculations involving hadron collisions with vector boson intermediate states simplify considerably. In the remainder of this paper we will work within the parton picture and the effective $W$ approximation.

The utility of this description is that a complex cross-section can be factorized into a sequence of simpler subprocesses [49, 50]. Dropping the contribution from $Z$ bosons, the effective cross-section for weak boson fusion can be written [47, 46]

$$
\sigma_{\text {eff }}=\int \frac{\mathrm{d} x_{1}}{x_{1}} \frac{\mathrm{d} x_{2}}{x_{2}} \sum_{\lambda \in \pm, L} \Gamma\left(W_{\lambda}^{+} W_{\lambda}^{-} \rightarrow h\right)_{x_{1} x_{2} s} F_{W_{\lambda}^{+}}\left(x_{1}\right) F_{W_{\lambda}^{-}}\left(x_{2}\right),
$$

where, $\pm L$ are transverse and longitudinal polarization modes, and $s$ is the centre of momentum energy of the collision. The functions $F(x)$ can be calculated or measured experimentally. Eq. (15) shows that the remaining chameleon corrections can be determined from the on-shell rate $\Gamma\left(W^{+} W^{-} \rightarrow h\right)$, taken to occur at centre of 
momentum energy $x_{1} x_{2} s$. In what follows, we will concentrate on modifications to this quantity.

\subsection{New physics and the $W^{+} W^{-} h, Z Z h$ vertices}

The rate $\Gamma(V \bar{V} \rightarrow h)$ depends on the amplitude of a Green's function describing effective $Z Z H$ and $W W H$ vertices. This Green's function captures the appearance of new physics in Eq. (15). In many theories of physics beyond the Standard Model, the form of the cubic and quartic vertices in Eq. (14) are unmodified. In these theories, corrections to the self-energy diagrams of $Z, W^{ \pm}, \gamma$ and $H$ particles disrupt tree-level relationships between the particle masses $\left\{M_{Z}, M_{W}, M_{H}\right\}$, the fine structure constant $\alpha$, and the Fermi constant $G_{F}$. This disruption can be summarized using oblique parameters introduced by Peskin \& Takeuchi [51, 52] and refined by Maksymyk, London \& Burgess [53, 54]. In our model there are both oblique and non-oblique corrections. To summarize our results we use the oblique notation of Refs. [53, 54] and note differences explicitly where they occur.

Consider the Green's function describing an effective $Z Z h$ vertex, from which the $W W h$ result can be derived after trivial modifications. The S-matrix element must depend on

$$
\mid\}_{Z}^{Z}-\left.h\right|^{2} \propto M_{Z}^{4} G_{F} \times \text { wavefunction renormalizations, }
$$

where the wavefunction renormalizations arise on transition to the $S$-matrix. We work in unitary gauge, where the Goldstone modes associated with the SU(2) Higgs doublet are absorbed as longitudinal polarizations of $Z$ and $W^{ \pm}$. Accounting for oblique corrections, the $Z$ propagator is

$$
\left\langle Z^{a}\left(k_{1}\right) Z^{b}\left(k_{2}\right)\right\rangle=-\mathrm{i}(2 \pi)^{4} \delta\left(k_{1}+k_{2}\right)\left(\eta^{a b}+\frac{k^{a} k^{b}}{M_{Z}^{2}}\right) \Delta^{\prime}\left(k^{2}\right),
$$

where $\Delta^{\prime}\left(k^{2}\right)$ takes the form

$$
\Delta^{\prime}\left(k^{2}\right)^{-1} \equiv k^{2}+M_{Z}^{2}-\Pi_{Z Z}\left(k^{2}\right) .
$$

In Eqs. (17), $k$ stands for either $k_{1}$ or $k_{2}$. The quantity $\Pi_{Z Z}$ is defined as follows. We choose $\mathrm{i} \Pi_{Z Z}^{a b}\left(k^{2}\right)$ to be the sum of all one-particle-irreducible graphs connecting an ingoing and an outgoing $Z$. In vacuum this has a unique tensorial decomposition,

$$
\Pi_{Z Z}^{a b}\left(k^{2}\right) \equiv \eta^{a b} \Pi_{Z Z}^{(0)}\left(k^{2}\right)+k^{a} k^{b} \Pi_{Z Z}^{(2)}\left(k^{2}\right) .
$$

We neglect the term involving $\Pi_{Z Z}^{(2)}$ and can therefore drop superscript ' 0 's without ambiguity, so that $\Pi_{Z Z}^{(0)} \rightarrow \Pi_{Z Z}$. It is this quantity which appears in Eq. (18). If the mass of external fermions is at most $\sim M_{f}$, this neglect is equivalent to dropping powers of $M_{f} / M_{Z}$. It is likely to be a good approximation provided the Higgs is not too 
heavy: for a Higgs lighter than the top mass, $M_{t} \simeq 173 \mathrm{GeV}$, decay into a top quark is kinematically forbidden. Therefore, $M_{f} / M$ is at most of order $10^{-1}$ to $10^{-2}$.

Including oblique corrections, the $Z$ mass becomes

$$
M_{Z}^{2}=\tilde{M}_{Z}^{2}\left(1-\frac{\Pi_{Z Z}\left(-M_{Z}^{2}\right)}{M_{Z}^{2}}\right) .
$$

A similar formula can be written for the $W$ propagator, making the replacements $M_{Z} \rightarrow M_{W}$ and $\Pi_{Z Z} \rightarrow \Pi_{W W}$. Quantities with a tilde, such as $\tilde{M}_{Z}$ and $\tilde{G}_{F}$, refer to the value of these parameters in the absence of oblique corrections. With the same conventions, the Fermi constant satisfies

$$
G_{F}=\tilde{G}_{F}\left(1+\frac{\Pi_{W W}(0)}{M_{W}^{2}}\right) .
$$

$G_{F}$ parametrizes the strength of the weak force near zero momentum transfer. Allowing for these shifts, the decay rate $\Gamma(Z Z \rightarrow h)$ is related to the pure Standard Model rate by the rule

$$
\begin{aligned}
\frac{\Gamma(Z Z \rightarrow h)}{\tilde{\Gamma}(Z Z \rightarrow h)} & =1+2 \frac{\Pi_{Z Z}\left(-M_{Z}^{2}\right)}{M_{Z}^{2}}-\frac{\Pi_{W W}(0)}{M_{W}^{2}}+2 \Pi_{Z Z}^{\prime}\left(-M_{Z}^{2}\right)+\Pi_{H H}^{\prime}\left(-M_{H}^{2}\right) \\
& =1+\alpha(2 V+R) .
\end{aligned}
$$

For $W$ bosons the decay rate is

$$
\begin{aligned}
\frac{\Gamma(W W \rightarrow h)}{\tilde{\Gamma}(W W \rightarrow h)} & =1+2 \frac{\Pi_{W W}\left(-M_{W}^{2}\right)}{M_{W}^{2}}-\frac{\Pi_{W W}(0)}{M_{W}^{2}}+2 \Pi_{W W}^{\prime}\left(-M_{W}^{2}\right)+\Pi_{H H}^{\prime}\left(-M_{H}^{2}\right) \\
& =1+\alpha(2 W+R),
\end{aligned}
$$

where, as above, $\alpha \approx 1 / 137$ is the fine structure constant. Eqs. (22) and (23) have been written in terms of the conventional oblique quantities $V$ and $W$, which are defined to satisfy $[53$

$$
\begin{aligned}
& \left.\alpha V \equiv \frac{\mathrm{d}}{\mathrm{d} k^{2}} \Pi_{Z Z}\left(k^{2}\right)\right|_{k^{2}=-M_{Z}^{2}}-\frac{\Pi_{Z Z}(0)-\Pi_{Z Z}\left(-M_{Z}^{2}\right)}{M_{Z}^{2}}, \\
& \left.\alpha W \equiv \frac{\mathrm{d}}{\mathrm{d} k^{2}} \Pi_{W W}\left(k^{2}\right)\right|_{k^{2}=-M_{W}^{2}}-\frac{\Pi_{W W}(0)-\Pi_{W W}\left(-M_{W}^{2}\right)}{M_{W}^{2}} .
\end{aligned}
$$

In addition, we have introduced a new quantity $R$ which is a measure of the Higgs' wavefunction renormalization,

$$
\left.\alpha R \equiv \frac{\mathrm{d}}{\mathrm{d} k^{2}} \Pi_{H H}\left(k^{2}\right)\right|_{k^{2}=-M_{H}^{2}}+\frac{\Pi_{Z Z}(0)}{M_{Z}^{2}} .
$$

If the dark energy coupling scale is greater than the typical scale of electroweak processes, $M_{\mathrm{EW}} \sim 1 \mathrm{TeV}$, we expect $V$ and $W$ to be negligible [25]. The impact of new physics is therefore contained entirely in $R$.

\section{Corrections from a dark energy scalar}

Eqs. (22) -(23) determine the sensitivity of weak boson fusion and Higgsstrahlung to new physics. This sensitivity is measured by the Higgs oblique parameter, $R$. In this section 
we make a quantitative estimate of its magnitude. To do so, we must be precise about the corrections $\Pi_{Z Z}$ and $\Pi_{W W}$ which modify the Standard Model prediction. In $\$ 4.1$ we determine these quantities in a low energy chameleon-type model coupled to the gauge bosons. We calculate oblique corrections to the production rate, and show that they are sensitive to the high energy completion of the theory. In $\$ 4.2$ we compute non-oblique corrections generated by integrating out heavy fermions. These are described by a new quartic coupling between the Higgs field and the gauge bosons.

\subsection{Oblique corrections in the low-energy theory}

A dark energy field induces both straight and oblique corrections to the vacuum polarizations of the Higgs and gauge bosons. In Ref. [25] it was argued that the straight corrections effectively divide into processes involving "chameleonstrahlung," where dark energy particles are produced but escape the detector, and a collection of "bridges," "daisies" and "rainbows" which dress the bare processes of the Standard Model. At leading order, these dressings are momentum independent. Chameleonstrahlung was shown to give constraints roughly comparable to those arising from oblique corrections. In this paper we focus on oblique corrections only.

After electroweak symmetry breaking, we can parameterize the interactions of Eq. (9) by adopting an effective $Z$ boson Lagrangian of the form employed in Ref. [25],

$S=-\frac{1}{4} \int \mathrm{d}^{4} x\left\{B(\beta \chi)\left(\partial^{a} Z^{b}-\partial^{b} Z^{a}\right)\left(\partial_{a} Z_{b}-\partial_{b} Z_{a}\right)+2 B_{H}\left(\beta_{H} \chi\right) M_{Z}^{2} Z^{a} Z_{a}\right\}$.

The functions $B$ and $B_{H}$ should satisfy $B(0)=B_{H}(0)=1$, but depend on the details of ultra-violet physics. More precisely, they are derived from Eq. (9) and similar higher-order diagrams involving more powers of $\delta \chi$. Likewise, the couplings $\beta$ and $\beta_{H}$ are inherited from whatever heavy particles are integrated out to generate this interaction. Working with a sharp momentum cutoff, the $Z$ vacuum polarization was determined in Ref. [25] and found to be

$$
\begin{aligned}
\Pi_{Z Z}\left(k^{2}\right)=\frac{\beta^{2}}{8 \pi^{2}} & \frac{\bar{B}^{\prime 2}}{\bar{B}} \int_{0}^{1} \mathrm{~d} x\left\{\frac{2 k^{2}+\gamma^{2} M_{Z}^{2}}{4}\left[\Lambda^{2}-\frac{\Lambda^{2}}{2} \frac{\Lambda^{2}}{\Lambda^{2}+\Sigma_{Z}^{2}}-\Sigma_{Z}^{2} \ln \left(1+\frac{\Lambda^{2}}{\Sigma_{Z}^{2}}\right)\right]\right. \\
+ & \left(x k^{2}+\gamma M_{Z}^{2}\right)^{2}\left[-\frac{1}{2} \frac{\Lambda^{2}}{\Lambda^{2}+\Sigma_{Z}^{2}}+\frac{1}{2} \ln \left(1+\frac{\Lambda^{2}}{\Sigma_{Z}^{2}}\right)\right] \\
& \left.-\frac{\Omega}{2}\left(k^{2}+\epsilon M_{Z}^{2}\right)\left[\frac{\Lambda^{2}}{2}-\frac{M_{\chi}^{2}}{2} \ln \left(1+\frac{\Lambda^{2}}{M_{\chi}^{2}}\right)\right]\right\},
\end{aligned}
$$

where $\Omega$ satisfies

$$
\Omega \equiv \frac{\bar{B}^{\prime \prime} \bar{B}}{\bar{B}^{\prime 2}}
$$

and $\bar{B} \equiv B(\beta \bar{\chi})$. The parameters $\epsilon$ and $\gamma$ are defined by

$$
\begin{aligned}
& \epsilon=\frac{B_{H}^{\prime \prime}}{B^{\prime \prime}} \frac{\beta_{H}^{2}}{\beta^{2}} \\
& \gamma=\frac{B_{H}^{\prime}}{B^{\prime}} \frac{\beta_{H}}{\beta} .
\end{aligned}
$$




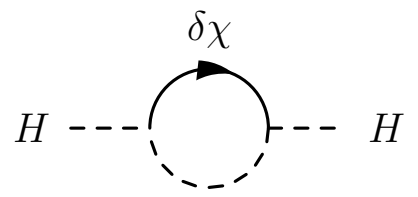

(a)

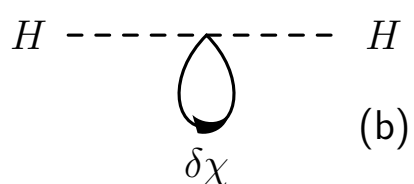

Figure 3. Processes contributing to the self-energy of the Higgs boson. An initial Higgs boson state, represented by a dashed line, radiates into scalar quanta $\chi$ (represented by a solid line) which are eventually re-absorbed to yield a final state characterized by the same quantum numbers and momentum as the initial state.

Also, $\Sigma_{Z}^{2}$ represents

$$
\Sigma_{Z}^{2} \equiv x(1-x) k^{2}+(1-x) M_{Z}^{2}+x M_{\chi}^{2},
$$

where $M_{\chi}$ is the mass of the dark energy fluctuation $\delta \chi$. Near $k^{2} \approx 0, \Pi_{Z Z}\left(k^{2}\right)$ has the approximate form

$$
\Pi_{Z Z}\left(k^{2}\right) \approx \frac{\beta_{H}^{2} \Lambda^{2}}{32 \pi^{2}} \frac{\bar{B}_{H}^{\prime 2}}{\bar{B}}\left(\frac{1}{2}-\frac{\bar{B}_{H}^{\prime \prime} \bar{B}}{\bar{B}_{H}^{\prime 2}}\right)+\mathrm{O}\left(\beta_{H}^{2} M_{\mathrm{EW}}^{2}\right) .
$$

We must determine the vacuum polarization of the Higgs. As above, we assume this to follow from a conformal coupling to $\chi$. In the Standard Model, this would give the coupling

$S \supseteq-\frac{1}{2} \int \mathrm{d}^{4} x\left\{B_{H}\left(\beta_{H} \chi\right)\left|\left(\partial_{a}+\mathrm{i} \vec{A}_{a} \cdot \vec{t}-\mathrm{i} B_{a} y\right) H\right|^{2}-C_{H}\left(\beta_{H} \chi\right) \mu^{2} H^{\dagger} H+\mathrm{O}\left(\left[H^{\dagger} H\right]^{2}\right)\right\}$,

where $\vec{A}$ and $B$ are the gauge fields of the unbroken $\mathrm{SU}(2)$ and $\mathrm{U}(1)_{Y}$ symmetries, respectively; $\vec{t}$ are a set of appropriately normalized generators of $\mathrm{SU}(2)$; and $y$ is the generator of $\mathrm{U}(1) . H$ is an $\mathrm{SU}(2)$ Higgs doublet, and $\mu$ is a standard parameter of the quartic Higgs potential, related to the Higgs mass by the rule $M_{H}^{2}=2 \mu^{2}$. In many models the phenomenological couplings $B, B_{H}$ and $C_{H}$ will be closely related, but for the present we leave them arbitrary. If no relationship exists between the couplings, we find that unitarity is not respected at tree level in two-body scattering of gauge bosons $\left[55,56,[57,58]\right.$ at energy scales above $\left[G_{F}\left|B(\beta \bar{\chi})-B_{H}\left(\beta_{H} \bar{\chi}\right)\right|\right]^{-1 / 2} \dagger$ In models containing more than one Higgs doublet we assume that Eq. (34) continues to give a good approximation to the couplings of the lightest neutral Higgs.

$\dagger$ Additional violations of perturbative unitarity near the chameleon scale $\beta^{-1}$ may arise from new dark energy exchange diagrams in two-body scattering. 
The Higgs vacuum polarization $\Pi_{H H}\left(k^{2}\right)$ can be computed. The one-loop contributions are shown in Fig. 3 , and depend on the following vertices:

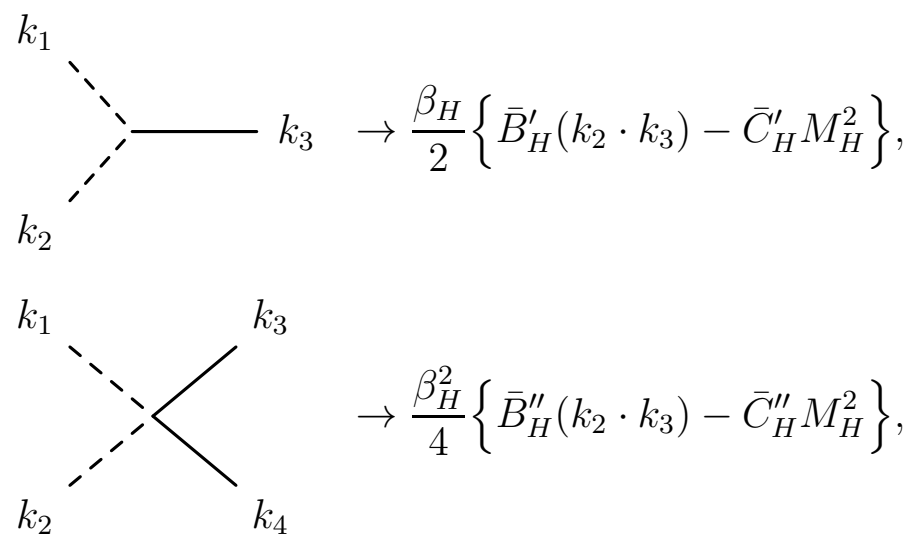

The diagram in Fig. 3(a) corresponds to a vacuum polarization

$$
\begin{aligned}
\Pi_{H H}\left(k^{2}\right)=\frac{\beta_{H}^{2}}{8 \pi^{2}} & \int_{0}^{1} \mathrm{~d} x \int_{0}^{\Lambda} \frac{\kappa^{3} \mathrm{~d} \kappa}{\left(\kappa^{2}+\Sigma_{H}^{2}\right)^{2}} \\
& \times\left[\bar{B}_{H}^{\prime 2}\left(\frac{k^{2} \kappa^{2}}{4}+x^{2} k^{4}\right)+\bar{C}_{H}^{\prime} M_{H}^{2}\left(\bar{C}_{H}^{\prime} M_{H}^{2}+2 \bar{B}_{H}^{\prime} k^{2} x\right)\right],
\end{aligned}
$$

where $x$ is a Feynman parameter and we have rotated to Euclidean signature. In analogy with Eq. (32), $\Sigma_{H}$ is defined so that

$$
\Sigma_{H}^{2}=x(1-x) k^{2}+(1-x) M_{H}^{2}+x M_{\chi}^{2} .
$$

The diagram in Fig. 3 (b) contributes

$$
\Pi_{H H}\left(k^{2}\right)=-\frac{\beta_{H}^{2}}{16 \pi^{2}} \int_{0}^{\Lambda} \frac{\kappa^{3} \mathrm{~d} \kappa}{\kappa^{2}+M_{\chi}^{2}}\left(\bar{B}_{H}^{\prime \prime} k^{2}+\bar{C}_{H}^{\prime \prime} M_{H}^{2}\right) .
$$

Carrying out the $\kappa$ integrals, we find

$$
\begin{aligned}
\Pi_{H H}\left(k^{2}\right)= & \frac{\beta_{H}^{2}}{8 \pi^{2}} \frac{1}{\bar{B}_{H}} \int_{0}^{1} \mathrm{~d} x\left\{\frac{\bar{B}_{H}^{\prime 2} k^{2}}{4}\left[\Lambda^{2}-\frac{\Lambda^{2}}{2} \frac{\Lambda^{2}}{\Lambda^{2}+\Sigma_{H}^{2}}-\Sigma_{H}^{2} \ln \left(1+\frac{\Lambda^{2}}{\Sigma_{H}^{2}}\right)\right]\right. \\
& +\left\{B_{H}^{\prime 2} x^{2} k^{4}+M_{H}^{2} \bar{C}_{H}^{\prime}\left(2 \bar{B}_{H}^{\prime} x k^{2}+M_{H}^{2} \bar{C}_{H}^{\prime}\right)\right\}\left[-\frac{1}{2} \frac{\Lambda^{2}}{\Lambda^{2}+\Sigma_{H}^{2}}+\frac{1}{2} \ln \left(1+\frac{\Lambda^{2}}{\Sigma_{H}^{2}}\right)\right] \\
& \left.-\frac{\bar{B}_{H}}{2}\left(\bar{B}_{H}^{\prime \prime} k^{2}+M_{H}^{2} \bar{C}_{H}^{\prime \prime}\right)\left[\frac{\Lambda^{2}}{2}-\frac{M_{\chi}^{2}}{2} \ln \left(1+\frac{\Lambda^{2}}{M_{\chi}^{2}}\right)\right]\right\} .
\end{aligned}
$$

From Eqs. (28) and (40) it is possible to compute $R$, the parameter which summarizes the oblique dependence of the rates $\Gamma(Z Z \rightarrow h)$ and $\Gamma\left(W^{+} W^{-} \rightarrow h\right)$. We find

$\alpha R=\frac{\beta_{H}^{2} \Lambda^{2}}{32 \pi^{2}} \frac{\bar{B}_{H}^{\prime 2}}{\bar{B}}\left[\frac{1}{2}\left(1+\frac{\bar{B}}{\bar{B}_{H}}\right)-2 \frac{\bar{B}_{H}^{\prime \prime} \bar{B}}{\bar{B}_{H}^{\prime 2}}\right]+$ finite terms of order $\mathrm{O}\left(\beta_{H}^{2} M_{\mathrm{EW}}^{2}\right)$.

At leading order in the divergence, it is independent of $C_{H}$. We note, however, that a dependence on $C_{H}$ persists among those terms which are finite in the limit $\Lambda \rightarrow \infty$. These finite terms are of order $\beta_{H}^{2} M_{\mathrm{EW}}^{2}$ and can be neglected when $\beta_{H} \ll(1 \mathrm{TeV})^{-1}$. We conclude that the oblique correction is very small, unless the divergent part of Eq. (41) can contribute a significant effect. 
What is the meaning of the divergent term in Eq. (41)? One must be wary when reasoning with power-law divergences, because they can be ascribed no invariant significance. For example, they are absent in dimensional regularization. In Ref. 25] it was found that similar divergences could be absorbed in renormalizations of $G_{F}$ and the vector boson masses, $M_{Z}$ and $M_{W}$. The divergence in Eq. (41) cannot be absorbed in this way. It expresses a sensitivity to whatever physics completes the low-energy theory containing the Standard Model and the effective interaction, Eq. (9)). It is not a prediction that large effects should be observed in a particle collider. The same divergence arises in all models with this low-energy limit, irrespective of what physics takes place at high energy. To fix its value with confidence, we must know the details of the high energy completion. In the next section we compute its value for a model in which the low energy theory is obtained by integrating out heavy fermions with SUSY-like couplings.

\subsection{Higgs couplings from heavy charged particles}

If it is obtained as the low energy effective theory of some complete UV physics, Eq. (9) will be accompanied by other interactions which cannot be neglected. The most important is a new contact interaction between gauge bosons and the Higgs field, and in what follows we estimate its effect on Higgs production. We use the size of the contribution to Higgs production from this straight corrections to estimate the size of the cutoff that controls corrections due to interactions with dark energy.

Gauge invariance constrains which operators can appear in the low-energy theory. In the minimal scenario we are considering, the Higgs field is in the fundamental 2 representation of $\mathrm{SU}(2)$. By construction, a field strength term such as $F^{a b} F_{a b}$ transforms in the adjoint representation. Therefore the lowest order non-trivial interaction with the Higgs must involve $H^{\dagger} H$, making $H^{\dagger} H \operatorname{Tr}\left(F^{a b} F_{a b}\right)$ a gauge invariant dimension-six operator.

If this operator is present in the low-energy effective theory, it will modify our

expectation for Higgs production. Accordingly, we must evaluate its coefficient. The prediction is model-dependent. Consider a minimal scenario, where the heavy charged particles are fermions which have vertices with the lightest neutral Higgs of the form

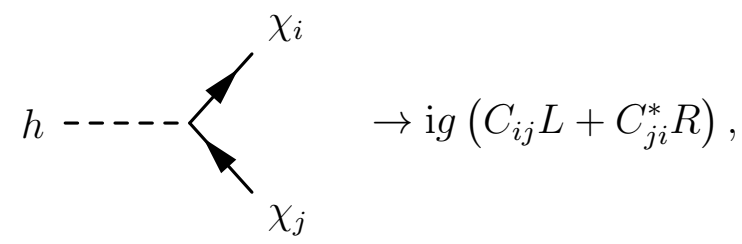

where $i$ and $j$ label different species of fermion and the strength of the coupling is parametrized by $g$. The operator $L \equiv\left(1+\gamma_{5}\right) / 2$ projects onto the left-chirality half of a Dirac spinor, and $R \equiv\left(1-\gamma_{5}\right) / 2$ is its conjugate. The $C_{i j}$ should be chosen real and symmetric if $\mathrm{CP}$ violation is to be avoided. 

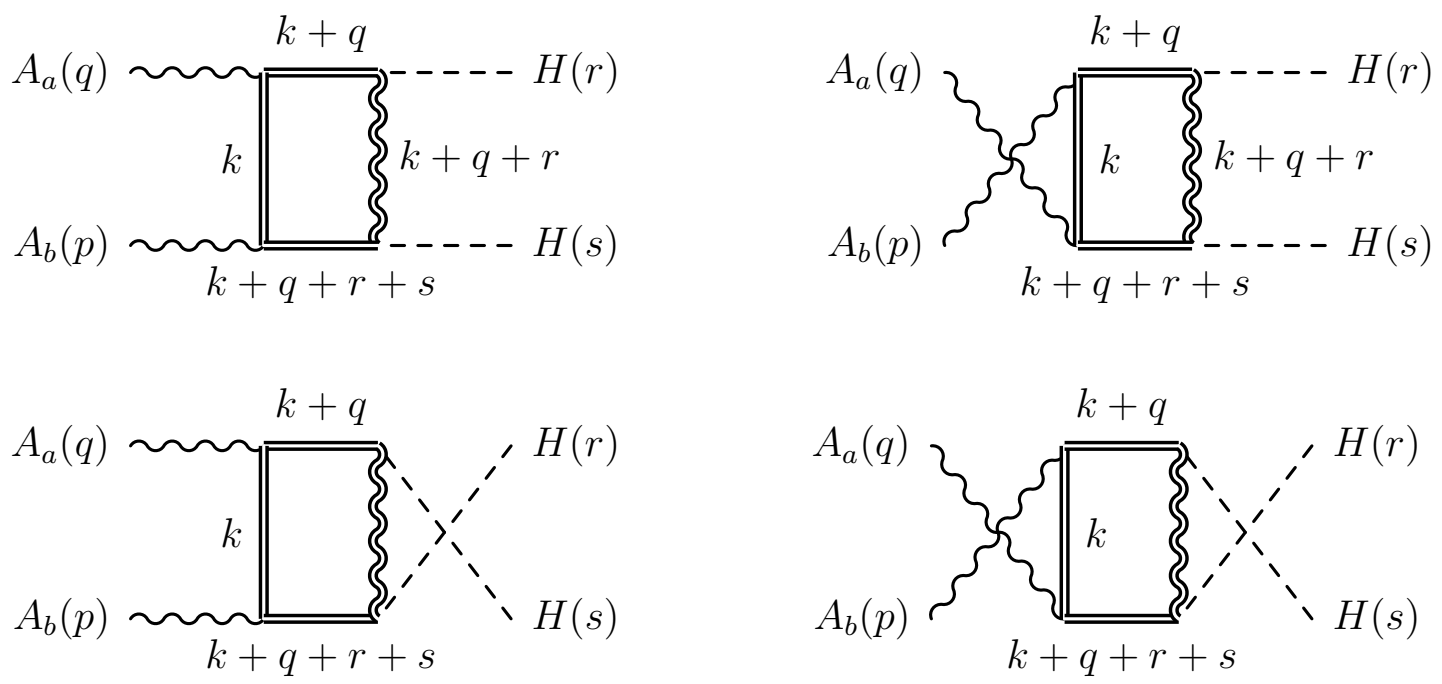

Figure 4. Leading interactions between the Higgs field and electroweak gauge bosons. The interactions are mediated by two species of chargino, $\lambda$ (straight doubled lines) and $\lambda^{\prime}$ (wiggly doubled lines), of masses $M$ and $M^{\prime}$, respectively.

In a supersymmetric Standard Model, the $\chi_{i}$ will be charginos and neutralinos. These have couplings to the lightest neutral Higgs of the form (42), with $C_{i j}$ determined by the various factors which diagonalize the chargino and neutralino mass matrices. Explicit expressions can be found in $\S A .7$ of Ref. [47]. In this case, the chargino and neutralino loops would be accompanied by heavy slepton loops which we do not calculate. There is no reason to expect the slepton contribution to be larger than the neutralino or chargino terms, so we anticipate that the fermion contribution alone is representative.

Eq. (42) gives rise to effective operators depicted in Fig. 4. We denote a fermion of species $i$ by a straight doubled line, and species $j$ by a wiggly doubled line. These diagrams must be summed over all $i$ and $j$. The correlation function $\left\langle A_{a}(q) A_{b}(p) h(r) h(s)\right\rangle$ satisfies

$$
\begin{aligned}
& \left\langle A_{a}(q) A_{b}(p) h(r) h(s)\right\rangle=(2 \pi)^{4} \delta(p+q+r+s) e^{2} g^{2} h(r) h(s) A_{a}(q) A_{b}(p) \int \frac{\mathrm{d}^{4} k}{(2 \pi)^{4}} \\
& \times \operatorname{tr} \sum_{i j}\left\{\gamma^{a} \frac{-\mathrm{i}(\not k+\not)+M_{i}}{(k+q)^{2}+M_{i}^{2}-\mathrm{i} \epsilon}\left(C_{i j} L+C_{j i}^{*} R\right) \frac{-\mathrm{i}(\not k+\not h+\not)+M_{j}}{(k+q+r)^{2}+M_{j}^{2}-\mathrm{i} \epsilon}\right. \\
& \left.\quad \times\left(C_{j i} L+C_{i j}^{*} R\right) \frac{-\mathrm{i}(\not k+\not l+\not+\not)+M_{i}}{(k+q+r+s)^{2}+M_{i}^{2}-\mathrm{i} \epsilon} \gamma^{b} \frac{-\mathrm{i} \not k+M_{i}}{k^{2}+M_{i}^{2}-\mathrm{i} \epsilon}\right\} \\
& +\left(\begin{array}{c}
p \leftrightarrow q \\
a \leftrightarrow b
\end{array}\right)+(r \leftrightarrow s),
\end{aligned}
$$

where $M_{i}$ is the mass of species $i$ and the symmetrizations are nested, so that $r \leftrightarrow s$ is carried out after the simultaneous exchanges $p \leftrightarrow q$ and $a \leftrightarrow b$. As above, the $k$ integral is divergent and potentially dependent on the labeling of momenta inside the loop, but once the integral has been regularized it can be checked that this dependence vanishes.

After a tedious calculation, we find that Eq. (43) can be reproduced using the 
following effective Lagrangian,

$$
\begin{aligned}
& L_{\text {eff }} \supseteq \sum_{i j} \frac{g^{2}}{3 !(4 \pi)^{2}} \frac{2}{18 M_{i}^{2}\left(1-x_{i j}^{2}\right)^{5}}\left\{e^{2} \zeta_{1}\left(x_{i j}\right) H^{\dagger} H \operatorname{Tr} F^{a b} F_{a b}+\zeta_{2}\left(x_{i j}\right)\left|D^{2} H\right|^{2}\right. \\
& \left.+\zeta_{3}\left(x_{i j}\right)\left(D_{a} D_{b} H\right)^{\dagger}\left(D^{a} D^{b} H\right)\right\}
\end{aligned}
$$

where $x_{i j} \equiv M_{j} / M_{i}$ and the functions $\left\{\zeta_{1}, \zeta_{2}, \zeta_{3}\right\}$ satisfy

$$
\begin{aligned}
\zeta_{1}\left(x_{i j}\right) \equiv & \Sigma_{i j}\left[8-49 x_{i j}^{2}+99 x_{i j}^{4}-71 x_{i j}^{6}+13 x_{i j}^{8}-12 x_{i j}^{4}\left(3-7 x_{i j}^{2}+2 x_{i j}^{4}\right) \ln x_{i j}\right] \\
& -2 x_{i j} \Gamma_{i j}\left[4-6 x_{i j}^{2}-9 x_{i j}^{4}+14 x_{i j}^{6}-3 x_{i j}^{8}+6 x_{i j}^{2}\left(3-6 x_{i j}^{2}+x_{i j}^{4}\right) \ln x_{i j}\right], \\
\zeta_{2}\left(x_{i j}\right) \equiv & 2 \Sigma_{i j}\left[7-47 x_{i j}^{2}+63 x_{i j}^{4}-25 x_{i j}^{6}+2 x_{i j}^{8}-12 x_{i j}^{4}\left(6-5 x_{i j}^{2}+x_{i j}^{4}\right) \ln x_{i j}\right] \\
& -4 x_{i j} \Gamma_{i j}\left[2+9 x_{i j}^{2}-18 x_{i j}^{4}+7 x_{i j}^{6}+6 x_{i j}^{2}\left(3-x_{i j}^{4}\right) \ln x_{i j}\right], \\
\zeta_{3}\left(x_{i j}\right) \equiv & -2 \Sigma_{i j}\left[4-23 x_{i j}^{2}+63 x_{i j}^{4}-49 x_{i j}^{6}+5 x_{i j}^{8}+12 x_{i j}^{6}\left(5-x_{i j}^{2}\right) \ln x_{i j}\right] \\
& +2 x_{i j} \Gamma_{i j}\left[1-9 x_{i j}^{2}-9 x_{i j}^{4}+17 x_{i j}^{6}-12 x_{i j}^{4}\left(3+x_{i j}^{2}\right) \ln x_{i j}\right] .
\end{aligned}
$$

The quantities $\Sigma_{i j}$ and $\Gamma_{i j}$ are defined by

$$
\begin{array}{ll}
\Sigma_{i j} \equiv C_{i j} C_{i j}^{*}+C_{j i} C_{j i}^{*} & \text { (no sum on } i \text { or } j \text { ) } \\
\Gamma_{i j} \equiv C_{i j} C_{j i}+C_{i j}^{*} C_{j i}^{*} & \text { (no sum on } i \text { or } j \text { ) }
\end{array}
$$

To exhibit its gauge invariance, Eq. (44) has been written in terms of a conventionally normalized $\mathrm{SU}(2)$ doublet $H$, which coincides with the field-space orientation of the lightest neutral Higgs. It will be accompanied by higher derivative terms, represented by $\mathrm{O}\left(\partial^{4}\right)$, which have been neglected. There is also a term of the form $c h^{2} A_{a} A^{a}$, with $c$ a divergent constant, whose role is to renormalize the charge $e$. We discard this term and take $e$ to be the renormalized charge. At higher order in $H$, arbitrary powers of $H^{\dagger} H$ may be generated. These will lead to higher-dimension operators which couple polynomials of $H$ and its derivatives to the gauge field, but at leading order we can restrict our attention to Eq. (44).

To determine the correct order of magnitude for $\Lambda$, the cutoff used in the calculation of the oblique corrections in $\S 4.1$, it is safest to match to whatever theory controls physics in the ultra-violet [59]. In the present case, this is summarized by Eq. (44). Using the effective interactions in Eq. (44) it is possible to compute the enhancement to Higgs production due to these straight corrections and the represent this in the form of an oblique correction. This will give an estimate of the size of the cut off needed in the calculation of the oblique corrections in $\$ 4.1$. As a reasonable approximation, we take the $\mathrm{SU}(2)$ doublet, $H$, to develop a vacuum expectation value of order the Standard Model scale, $G_{F}^{-1 / 2}$. We take neutral excitations around this condensate to be representative of the interaction with the lightest neutral Higgs. Choosing the gauge field to be the Abelian vector associated with the $Z$, the resulting effective Lagrangian is

$$
\begin{aligned}
& L_{\text {eff }} \supseteq \frac{e^{2} g^{2}}{2592 \pi^{2}} \sum_{i j} \frac{\left(\sqrt{2} G_{F}\right)^{-1}}{M_{i}^{2}\left(1-x_{i j}^{2}\right)^{5}}\left\{\left[2 \zeta_{1}\left(x_{i j}\right)+2 \zeta_{3}\left(x_{i j}\right)\right] h\left(\partial_{a} Z_{a} \partial^{a} Z^{b}-\partial_{a} Z_{b} \partial^{b} Z^{a}\right)\right. \\
& \left.-\left[\zeta_{2}\left(x_{i j}\right)+\zeta_{3}\left(x_{i j}\right)\right] \partial^{2} h Z^{a} Z_{a}+\zeta_{3}\left(x_{i j}\right) h Z^{b} \partial^{2} Z_{b}\right\} .
\end{aligned}
$$


In the limit where the relative velocity of the colliding vector bosons goes to zero $₫$ and defining

$$
\hat{\zeta}_{m} \equiv \sum_{i j} \frac{\zeta_{m}\left(x_{i j}\right)}{M_{i}^{2}\left(1-x_{i j}^{2}\right)^{5}}
$$

we find

$$
\frac{\delta \Gamma(Z Z \rightarrow h)}{\Gamma(Z Z \rightarrow h)}=\left(\frac{e^{2} g^{2}}{2592 \sqrt{3} \pi^{2} G_{F}}\right)^{2}\left\{28 \hat{\zeta}_{1}^{2}-32 \hat{\zeta}_{1} \hat{\zeta}_{2}-28 \hat{\zeta}_{1} \hat{\zeta}_{3}+25 \hat{\zeta}_{2} \hat{\zeta}_{3}+35 \hat{\zeta}_{3}^{2}\right\}
$$

Despite appearances Eq. (52) is dimensionless, because each $\hat{\zeta}_{i}$ has the same dimension as $G_{F}$, [mass $]^{-2}$. The magnitude of this correction varies with the mass ratio, $x_{i j}$, approaching zero as $x_{i j} \rightarrow 1$ but asymptoting to an approximate constant for large or small ratios. In a typical supersymmetric Standard Model the chargino and neutralino masses are undetermined, but provided there is not total mass degeneracy we expect that this threshold correction generates a contribution represented by a cutoff of order

$$
\Lambda \simeq \beta_{H}^{-1} \times \frac{e^{2} g^{2}}{M^{2} G_{F}} .
$$

This is typically a rather small number, leading to an essentially negligible enhancement in the Higgs production rate.

\section{Conclusions}

In this paper we have derived the low energy effective theory which governs interactions between the gauge bosons of the electroweak sector and a dark energy scalar field. The dark energy is taken to have conformal couplings to the matter species of the Standard Model. It is possible that couplings of this type allow so-called "chameleon" behaviour, in which the field dynamically adjusts its mass to be large in regions of high average density, and small elsewhere. If such theories exist then they would lead to an unambiguous prediction of light dark energy quanta interacting in the beam pipe of any particle accelerator. Our low energy theory applies strictly in any model containing heavy charged fermions, but a very similar effective Lagrangian would apply for a model containing heavy charged particles of any spin. As a specific example, any supersymmetric Standard Model must contain charginos and neutralinos. These carry the quantum numbers of the $\mathrm{SU}(2) \times \mathrm{U}(1)$ gauge group and have masses of order the supersymmetry-breaking scale. However, our calculation is not restricted to the supersymmetric case.

One might worry that the presence of chameleonic quanta would change our predictions for the outcome of particle physics experiments. In Ref. [25] we argued this

$\ddagger$ The calculation does not need to be restricted to this kinematic limit, but it leads to simpler final expressions. Since the vector bosons are taken to be on-shell in the effective $W$ approximation, the invariant magnitude of any momenta will be of order $\sim M_{W}$. This implies that although the result may be modified by factors of $\mathrm{O}(1)$, it is unlikely that we commit a gross error by specializing to the zero-velocity limit. 
did not happen for any process without Higgs quanta. In this paper we have extended our argument to include Higgs production. In particular, using the low energy theory we have computed the oblique corrections to each of the Higgs, $Z$ and $W^{ \pm}$propagators at energies below the mass, $M$, of the heavy charged fermions. Such corrections modify the rates $\Gamma(Z Z \rightarrow h)$ and $\Gamma\left(W^{+} W^{-} \rightarrow h\right)$, where $h$ is the lightest neutral Higgs, and in principle could change the rate of production of this particle at a hadron collider. We find that the corrections diverge quadratically with the scale chosen as the cut off for the effective theory. This does not predict a large enhancement to the production of Higgs bosons from interactions with dark energy, instead indicating that the interactions with dark energy make this process sensitive to the UV physics.

Other contributions exist, generated by processes taking place at high energy, which are integrated out of the low-energy description. We determine these "threshold corrections" by integrating out heavy fermion loops which mediate interactions between the lightest neutral Higgs and the gauge bosons. The scale of these corrections can then be reinterpreted as a cutoff in an oblique calculation, of order $\Lambda \sim \beta_{H}^{-1} e^{2} g^{2} / M^{2} G_{F}^{2}$, and therefore leads to at most small effects. When $M$ is much larger than the Standard Model scale $G_{F}^{-1 / 2}$ it is entirely negligible. In an unconstrained theory, we might have anticipated a cutoff of order $\Lambda \sim \beta_{H}^{-1}$, because at this scale the effective dark energy theory becomes invalid. If this were true, it would be possible to contemplate corrections to the Higgs production rate of $\mathrm{O}(1)$ or larger, which would lie within the discovery reach of the LHC.

Unfortunately, the corrections are much smaller. Large terms could only arise from the relevant operator $H Z^{a} Z_{a}$, but its appearance is forbidden by gauge invariance above the scale of electroweak symmetry breaking. Therefore, we expect the coefficient of this term to be at most $G_{F}^{-1 / 2}$, rather than $\beta_{H}^{-1}$. Instead, the leading correction comes from the operators $H^{\dagger} H \operatorname{Tr} F^{a b} F_{a b},\left|D^{2} H\right|^{2}$ and $\left(D_{a} D_{b} H\right)^{\dagger}\left(D^{a} D^{b} H\right)$. These are dimension-six operators, because of the $\mathrm{SU}(2)$ nature of the Higgs doublet. Accordingly the cutoff is suppressed by $\left(M^{2} G_{F}\right)^{-1}$, making it small. This must be typical of any UV correction because there is no relevant operator we can write down which will couple $\chi$ to the gauge fields. To get a larger effect, it appears to be necessary to break the gauge invariance of the theory. This does not rule out the possibility that dark energy could be responsible for an enhancement in the Higgs production rate, but such a scenario would apparently require exotic physics.

In the context of particle colliders where strong magnetic fields are present, the coupling derived in $\$ 2$ implies a coupling of the chameleon to synchrotron radiation. This would lead to emergence of chameleon-like particles due to the Primakov effect. For most chameleon theories, the large mass assumed by chameleon-like particles in a dense environment implies that the beam pipe acts as a reflecting wall, preventing dark energy particles from leaking out. Hence collider experiments would actually take place in a dark energy bath. The analysis of this phenomenon is left for future work. 


\section{Acknowledgments}

We would like to thank Ben Allanach, Neil Barnaby, Cliff Burgess, Douglas Shaw and James Stirling for helpful discussions. CB is supported by the German Science Foundation (DFG) under the Collaborative Research Centre (SFB) 676. ACD and DS

are supported by STFC and the Cambridge Centre for Theoretical Cosmology. AW is supported by the Cambridge Centre for Theoretical Cosmology.

\section{References}

[1] S. Weinberg, The cosmological constant problem, Rev. Mod. Phys. 61 (1989) 1-23.

[2] B. Ratra and P. J. E. Peebles, Cosmological Consequences of a Rolling Homogeneous Scalar Field, Phys. Rev. D37 (1988) 3406.

[3] C. Wetterich, The Cosmon model for an asymptotically vanishing time dependent cosmological 'constant', Astron. Astrophys. 301 (1995) 321-328, arXiv:hep-th/9408025.

[4] I. Zlatev, L.-M. Wang, and P. J. Steinhardt, Quintessence, Cosmic Coincidence, and the Cosmological Constant, Phys. Rev. Lett. 82 (1999) 896-899, arXiv:astro-ph/9807002.

[5] S. M. Carroll, Quintessence and the rest of the world, Phys. Rev. Lett. 81 (1998) 3067-3070, arXiv:astro-ph/9806099.

[6] C. M. Will, The confrontation between general relativity and experiment, Living Rev. Rel. 4 (2001) 4, arXiv:gr-qc/0103036.

[7] J. Khoury and A. Weltman, Chameleon fields: Awaiting surprises for tests of gravity in space, Phys. Rev. Lett. 93 (2004) 171104, arXiv:astro-ph/0309300.

[8] J. Khoury and A. Weltman, Chameleon cosmology, Phys. Rev. D69 (2004) 044026, arXiv:astro-ph/0309411.

[9] D. F. Mota and J. D. Barrow, Local and Global Variations of The Fine Structure Constant, Mon. Not. Roy. Astron. Soc. 349 (2004) 291, arXiv:astro-ph/0309273.

[10] T. Clifton, D. F. Mota, and J. D. Barrow, Inhomogeneous gravity, Mon. Not. Roy. Astron. Soc. 358 (2005) 601, arXiv:gr-qc/0406001.

[11] D. F. Mota and D. J. Shaw, Evading equivalence principle violations, astrophysical and cosmological constraints in scalar field theories with a strong coupling to matter, Phys. Rev. D75 (2007) 063501, arXiv:hep-ph/0608078.

[12] W. Hu and I. Sawicki, Models of $f(R)$ Cosmic Acceleration that Evade Solar-System Tests, Phys. Rev. D76 (2007) 064004, arXiv:0705.1158.

[13] P. Brax, C. van de Bruck, A.-C. Davis, and D. J. Shaw, $f(R)$ Gravity and Chameleon Theories, Phys. Rev. D78 (2008) 104021, arXiv:0806.3415.

[14] P. Brax, C. van de Bruck, A.-C. Davis, J. Khoury, and A. Weltman, Detecting dark energy in orbit: The cosmological chameleon, Phys. Rev. D70 (2004) 123518, arXiv:astro-ph/0408415.

[15] P. Brax, C. Vandebruck, and A. C. Davis, A Chameleon Primer, arXiv:0706.1024.

[16] A. Weltman, Testing Chameleon models in the laboratory, in Conference proceedings of Rencontres de Moriond, The Gioi Publishers, 2008. arXiv:0805.3461.

[17] A. Weltman, Hunting for Chameleons in ALP searches, in Proceedings of the 4th Patras Workshop on Axions, Wimps and Wisps, 2008. arXiv:0809.4293. ISBN 978-3-935702-26-3.

[18] C. Burrage, Searching for axion-like-particles in the sky, Nuclear Physics B - Proceedings Supplements 194 (2009) 190-195. New Horizons for Modern Cosmology: Proceedings of the Galileo Galilei Institute Conferences on Dark Matter and Dark Energy.

[19] P. Brax, C. van de Bruck, A.-C. Davis, and D. Shaw, Laboratory Tests of Chameleon Models, arXiv:0911.1086.

[20] A. S. Chou et al., A Search for chameleon particles using a photon regeneration technique, Phys. Rev. Lett. 102 (2009) 030402, arXiv:0806.2438. 
[21] A. Upadhye, J. H. Steffen, and A. Weltman, Constraining chameleon field theories using the GammeV afterglow experiments, Phys. Rev. D81 (2010) 015013, arXiv:0911.3906.

[22] C. F. Kolda and D. H. Lyth, Quintessential difficulties, Phys. Lett. B458 (1999) 197-201, arXiv:hep-ph/9811375.

[23] R. D. Peccei, Light scalars in cosmology, arXiv:hep-ph/0009030.

[24] M. Doran and J. Jaeckel, Loop corrections to scalar quintessence potentials, Phys. Rev. D66 (2002) 043519, arXiv:astro-ph/0203018.

[25] P. Brax, C. Burrage, A.-C. Davis, D. Seery, and A. Weltman, Collider constraints on interactions of dark energy with the Standard Model, JHEP 09 (2009) 128, arXiv:0904.3002.

[26] M. A. Luty, M. Porrati, and R. Rattazzi, Strong interactions and stability in the DGP model, JHEP 09 (2003) 029, arXiv:hep-th/0303116.

[27] A. Nicolis, R. Rattazzi, and E. Trincherini, The galileon as a local modification of gravity, Phys. Rev. D79 (2009) 064036, arXiv:0811.2197.

[28] P. Brax, C. van de Bruck, A.-C. Davis, D. F. Mota, and D. J. Shaw, Testing Chameleon Theories with Light Propagating through a Magnetic Field, Phys. Rev. D76 (2007) 085010, arXiv:0707.2801.

[29] P. Brax, C. van de Bruck, and A.-C. Davis, Compatibility of the chameleon-field model with fifthforce experiments, cosmology, and PVLAS and CAST results, Phys. Rev. Lett. 99 (2007) 121103, arXiv:hep-ph/0703243.

[30] A. Mirizzi, G. G. Raffelt, and P. D. Serpico, Photon-axion conversion as a mechanism for supernova dimming: Limits from CMB spectral distortion, Phys. Rev. D72 (2005) 023501, arXiv:astro-ph/0506078.

[31] H. Gies, D. F. Mota, and D. J. Shaw, Hidden in the Light: Magnetically Induced Afterglow from Trapped Chameleon Fields, Phys. Rev. D77 (2008) 025016, arXiv:0710.1556.

[32] M. Ahlers, A. Lindner, A. Ringwald, L. Schrempp, and C. Weniger, Alpenglow - A Signature for Chameleons in Axion-Like Particle Search Experiments, Phys. Rev. D77 (2008) 015018, arXiv:0710.1555.

[33] P. Minkowski, $\mu \rightarrow$ ey at a Rate of One Out of 1-Billion Muon Decays?, Phys. Lett. B67 (1977) 421.

[34] M. Gell-Mann, P. Ramond, and R. Slansky, Complex spinors and unified theories, in Supergravity (D. Z. Freedman, ed.). North Holland, 1979. Print-80-0576 (CERN).

[35] T. Yanagida, Horizontal gauge symmetry and masses of neutrinos. In Proceedings of the Workshop on the Baryon Number of the Universe and Unified Theories, Tsukuba, Japan, 13-14 Feb 1979.

[36] P. Binetruy, Supersymmetry: Theory, experiment and cosmology. Oxford, UK: Oxford Univ. Pr. (2006) $520 \mathrm{p}$.

[37] C. Burrage, A.-C. Davis, and D. J. Shaw, Detecting Chameleons: The Astronomical Polarization Produced by Chameleon-like Scalar Fields, Phys. Rev. D79 (2009) 044028, arXiv:0809.1763.

[38] K. Van Bibber, N. R. Dagdeviren, S. E. Koonin, A. K. Kerman, and H. N. Nelson, Proposed experiment to produce and detect light pseudoscalars, Phys. Rev. Lett. 59 (Aug, 1987) 759-762.

[39] A. Afanasev et al., New Experimental limit on Optical Photon Coupling to Neutral, Scalar Bosons, Phys. Rev. Lett. 101 (2008) 120401, arXiv:0806.2631.

[40] C. Burrage, Supernova Brightening from Chameleon-Photon Mixing, Phys. Rev. D77 (2008) 043009, arXiv:0711.2966.

[41] A.-C. Davis, C. A. O. Schelpe, and D. J. Shaw, The Effect of a Chameleon Scalar Field on the Cosmic Microwave Background, Phys. Rev. D80 (2009) 064016, arXiv:0907.2672.

[42] C. A. O. Schelpe, Chameleon-Photon Mixing in a Primordial Magnetic Field, arXiv:1003.0232.

[43] C. Burrage, A.-C. Davis, and D. J. Shaw, Active Galactic Nuclei Shed Light on Axion-likeParticles, Phys. Rev. Lett. 102 (2009) 201101, arXiv:0902.2320.

[44] S. Weinberg, A Model of Leptons, Phys. Rev. Lett. 19 (1967) 1264-1266.

[45] Particle Data Group Collaboration, C. Amsler et al., Review of particle physics, Phys. Lett. 
B667 (2008) 1.

[46] W. Kilian, Electroweak symmetry breaking: The bottom-up approach, Springer Tracts Mod. Phys. 198 (2003) 1-113.

[47] J. F. Gunion, H. E. Haber, G. L. Kane, and S. Dawson, The Higgs Hunter's Guide. SCIPP-89/13.

[48] J. C. Collins and J.-w. Qiu, A new derivation of the Altarelli-Parisi equations, Phys. Rev. D39 (1989) 1398.

[49] J. Lindfors, Distribution functions for heavy vector bosons inside colliding particle beams, Z. Phys. C28 (1985) 427.

[50] P. W. Johnson, F. I. Olness, and W.-K. Tung, The effective vector boson method for high-energy collisions, Phys. Rev. D36 (1987) 291.

[51] M. E. Peskin and T. Takeuchi, A New constraint on a strongly interacting Higgs sector, Phys. Rev. Lett. 65 (1990) 964-967.

[52] M. E. Peskin and T. Takeuchi, Estimation of oblique electroweak corrections, Phys. Rev. D46 (1992) 381-409.

[53] I. Maksymyk, C. P. Burgess, and D. London, Beyond S, T and U, Phys. Rev. D50 (1994) 529-535, arXiv:hep-ph/9306267.

[54] C. P. Burgess, S. Godfrey, H. Konig, D. London, and I. Maksymyk, A Global fit to extended oblique parameters, Phys. Lett. B326 (1994) 276-281, [arXiv:hep-ph/9307337].

[55] C. H. Llewellyn Smith, High-Energy Behavior and Gauge Symmetry, Phys. Lett. B46 (1973) $233-236$.

[56] D. A. Dicus and V. S. Mathur, Upper bounds on the values of masses in unified gauge theories, Phys. Rev. D7 (1973) 3111-3114.

[57] J. M. Cornwall, D. N. Levin, and G. Tiktopoulos, Derivation of Gauge Invariance from High-Energy Unitarity Bounds on the s Matrix, Phys. Rev. D10 (1974) 1145.

[58] J. M. Cornwall, D. N. Levin, and G. Tiktopoulos, Uniqueness of spontaneously broken gauge theories, Phys. Rev. Lett. 30 (1973) 1268-1270.

[59] C. P. Burgess and D. London, Uses and abuses of effective Lagrangians, Phys. Rev. D48 (1993) 4337-4351, arXiv:hep-ph/9203216. 